\title{
The study of the dynamics of the spacecraft landing on a celestial body with microgravity under different fixing conditions
}

\author{
Chen Danhe and Vsevolod V. Koryanov \\ Bauman Moscow State Technical University, Dynamics and flight control of motion of rockets and spacecraft Department, 105005, Moscow, \\ Russian Federation
}

\begin{abstract}
The features of angular motion of the spacecraft when landing on the surface of a small celestial body are studied. Different variants of the spacecraft landing using clamping engines and cable are proposed and the definition of the parameters of the landing system works using a fixing system under different conditions. Optimal conditions are recommended for securing the space lander on the surface of a small celestial body.
\end{abstract}

\section{Introduction}

Exploration of the small celestial body (asteroids, comets, act.) is one of the important space activities in recent years and it is carries out by launching the spacecraft to the small celestial bodies. In previous works of authors has been proposed the method determining the longitudinal motion parameters of spacecraft during landing on the surface of a small celestial body [2], and the method of calculation of dynamics of the angular movement while spacecraft landing on the surface of the celestial body with small gravitational field $[3,4]$.These methods are developed on the base of tripod lander with influence of clamping engines and cable tension, in order to secure the space lander on the surface of small celestial body $[6,7]$. The structure of lander and vector of forces acting on the lander are shown in picture 1,2 . Where, the OXYZ is the surface coordinate system (UCS) - X, Y, Z, is associated with the surface of the celestial body ( $X$ and $\mathrm{Z}$ axes lie in the plane of the landing surface, the $\mathrm{X}$ axis lies in the plane of the first gear).

\section{Modelling}

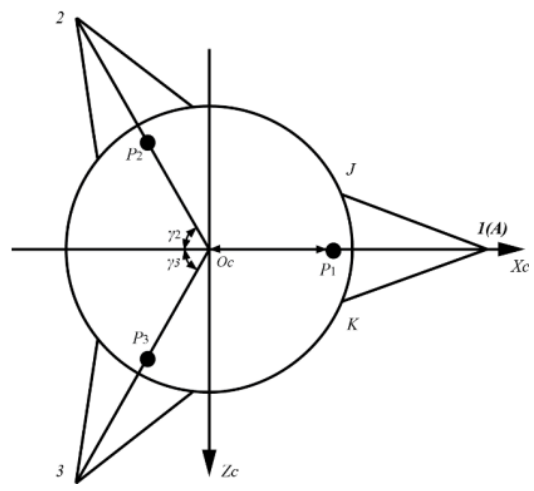

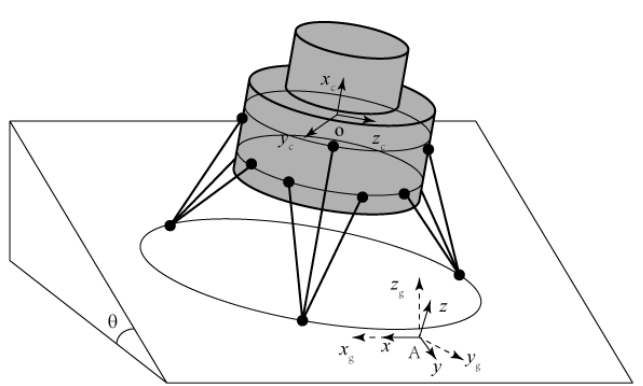

Figure 2. View of lander on the surface of celestial body

In Fig. 1: P1, P2, P3 - where fixed clamping engines; $\theta$-slope surface. The differential equation of the spatial movement of lander while landing on the surface of planet is described as below:

$$
\begin{aligned}
& m \frac{d V_{x}}{d t}=\sum_{j=1}^{n} F_{T x j}+T_{x}+P_{x}+m g_{x} \\
& m \frac{d V_{y}}{d t}=\sum_{j=1}^{n} F_{N j}+T_{y}+P_{y}+m g_{y} \\
& m \frac{d V_{z}}{d t}=\sum_{j=1}^{n} F_{T z j}+T_{z}+P_{z}+m g_{z} \\
& \frac{d x}{d t}=V_{x}, \frac{d y}{d t}=V_{y}, \frac{d z}{d t}=V_{z} \\
& J_{x} \frac{d \omega_{x}}{d t}=\sum_{j=1}^{n} M_{x c j}+M_{T c x}+\left(J_{y}-J_{z}\right) \omega_{y} \omega_{z} \\
& J_{y} \frac{d \omega_{y}}{d t}=\sum_{j=1}^{n} M_{y c j}+M_{T c y}+\left(J_{z}-J_{x}\right) \omega_{x} \omega_{z} \\
& J_{z} \frac{d \omega_{z}}{d t}=\sum_{j=1}^{n} M_{z c j}+M_{T c z}+\left(J_{x}-J_{y}\right) \omega_{y} \omega_{x}
\end{aligned}
$$

Figure 1. The structure of lander in $O X c Z c$ 


$$
\begin{aligned}
& \dot{\theta}=\sin \gamma \omega_{y}+\cos \gamma \omega_{z} \\
& \dot{\varphi}=\frac{\cos \gamma \omega_{y}-\sin \gamma \omega_{z}}{\cos \varphi} \\
& \dot{\gamma}=\omega_{x}-\frac{\cos \gamma \sin \varphi \omega_{y}-\sin \gamma \sin \varphi \omega_{z}}{\cos \varphi}
\end{aligned}
$$

where, $F_{T x}, F_{N}, F_{T z}$-impact on the gears when contacting with the surface; $T_{x}, T_{y}, T_{z}$ - cable tension; $P_{x}, P_{y}, P_{z}$ clamping force of the engines; $m g_{x}, m g_{y}, m g_{z}$ - projections of gravity on OXYZ axis; $V_{x}, V_{y}, V_{z}$ - the projections of the velocity vector of the CPA on $O X Y Z$ axis; $x, y, z-$ coordinates of the cm CPA; $,, \psi, \gamma$ - Euler angles of lander.

In the equations (1) considered two modes of fixing for lander while landing on the surface of celestial body, cable with specific anchor-penetrator, and clamping engines. Equation (1) can be solved by the following initial landing conditions for analyzing the characteristic of dynamical movement of space lander and the influences of fixing on it.

\subsection{Analysis of influence of clamping engines}

Consider different cases using clamping engines that are installed on the space lander. Clamping engines are switched under different conditions. Note that the number $\mathrm{NV}$ - embodiment when power is pressing, $\mathrm{NV}=1$ : the motors are switched on when any one leg of lander touches the surface; $\mathrm{NV}=2$ : the motors are turned on when any two legs touch the surface; $\mathrm{NV}=3$ : engines activated when the rate of the space lander on the OY axis is positive. The traction of clamping engines is $\mathrm{P}=$ $200 \mathrm{~N}$ and cable tension force $\mathrm{T}=0 \mathrm{~N}$, the results of the calculations are shown in the following figures.
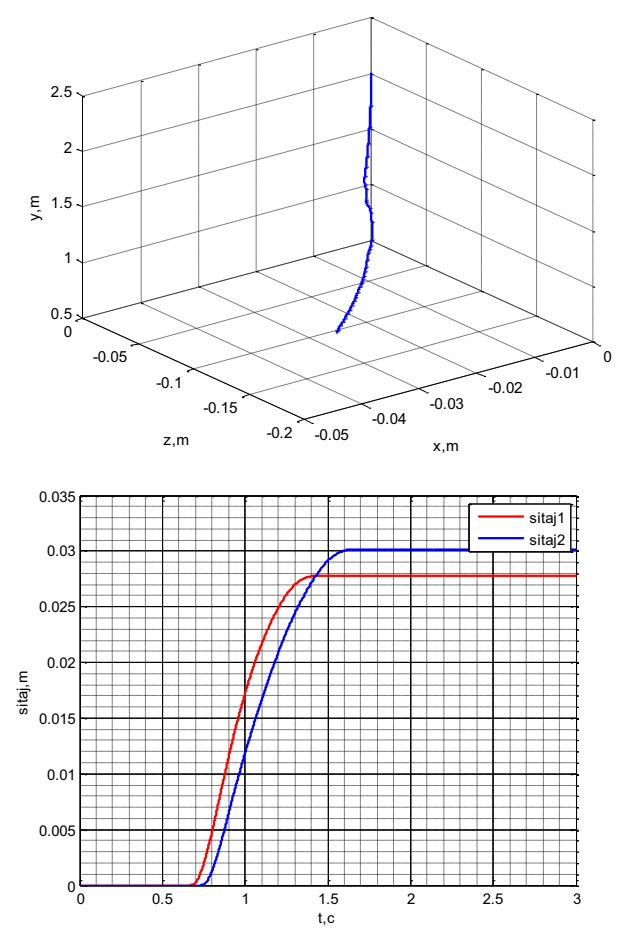

Figure 3. Engines operate under option $\mathrm{NV}=1$
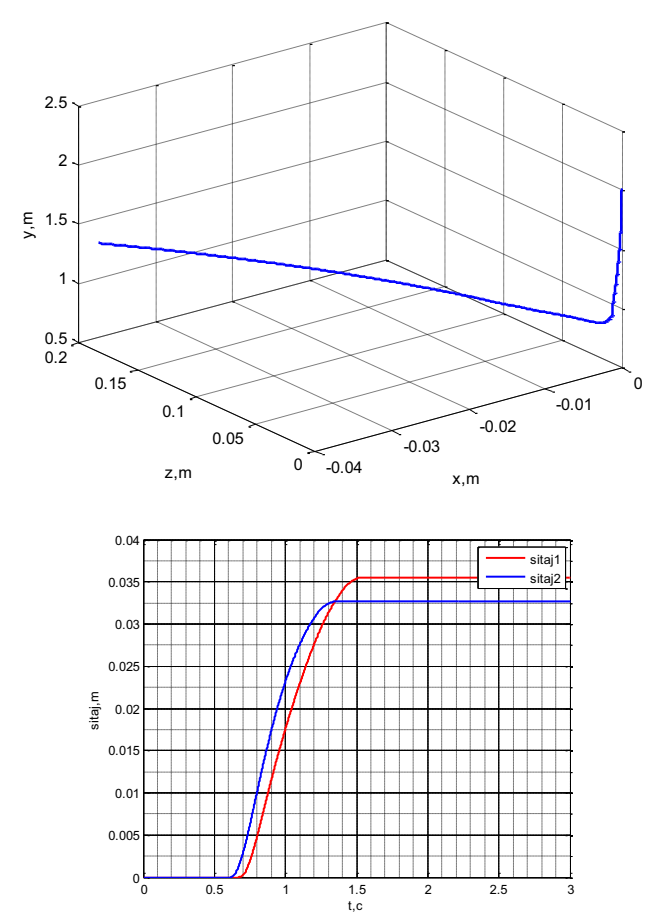

Figure 4. Engines operate under option $\mathrm{NV}=2$
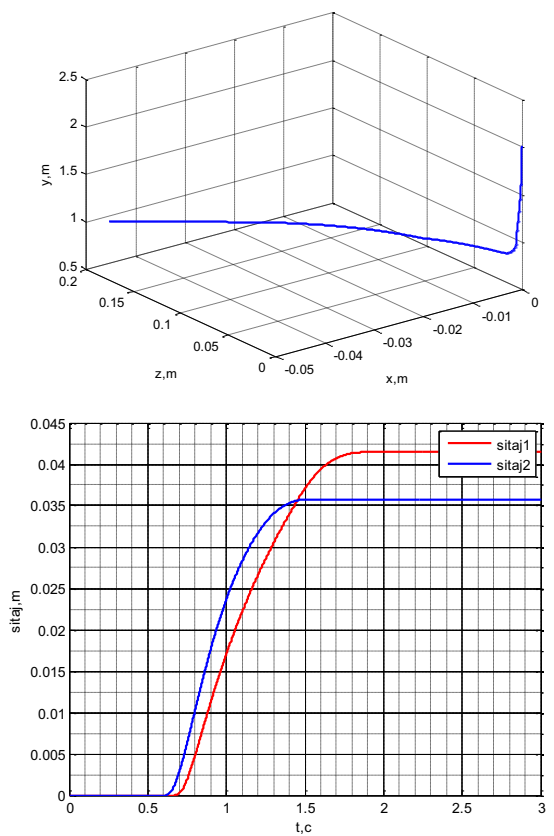

Figure 5. Engines operate under option $\mathrm{NV}=3$

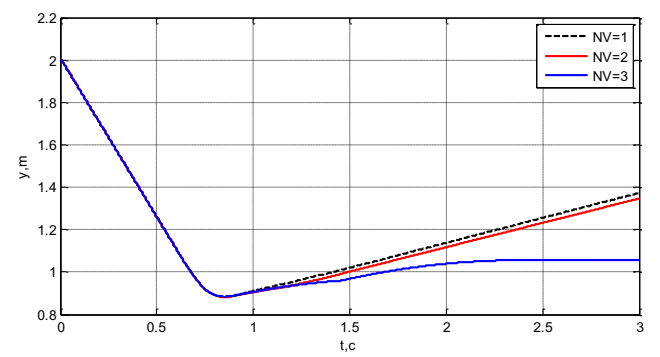

Figure 6. Comparison of the characteristics of the vertical position of space lander 


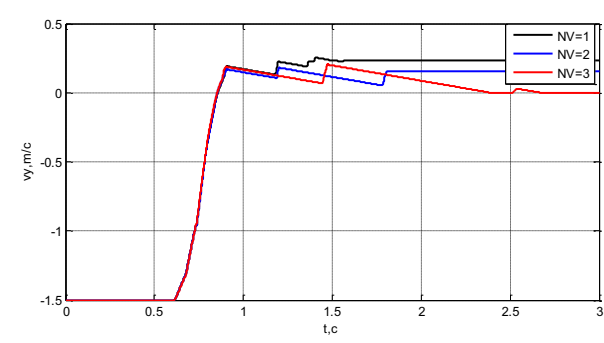

Figure 7. Comparison of the vertical velocity characteristics of space lander

The results of calculation results show that landing with the use of clamping engines with a certain tractive force could not afford to provide a secure fixation. Among the three modes clamping engines, which switched on if the space lander obtain positive vertical velocity, have greater stability to ensure a successful landing, compared with other options.

\subsection{Analysis of influence of cable tension}

Landing on the surface of a small celestial body using the clamping engines always contains limitation. At the same time, this study shows the use of special device - Anchorpenetrator, which shoot into the ground surface of a celestial body on landing and is given constant force tension down.
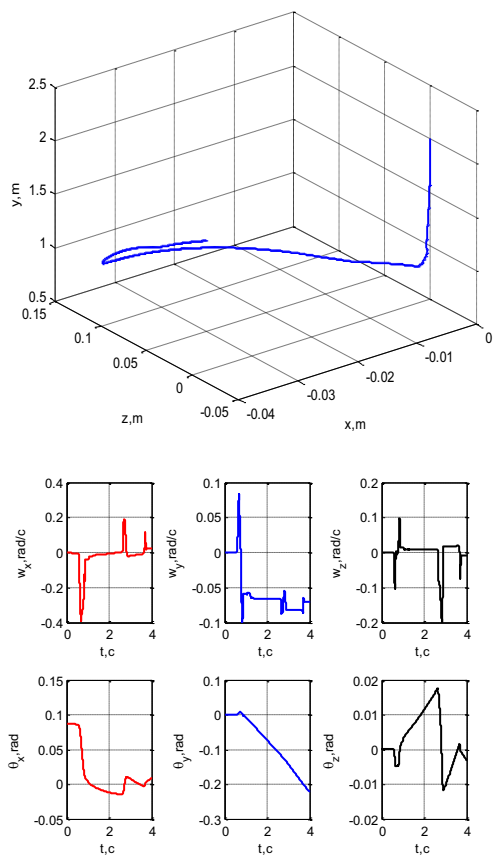

Figure 8. The cable works for all landing time, $T=200 \mathrm{~N}, \mathrm{P}=$ $200 \mathrm{~N}$

The value of the power cable tension is given by the calculation program, which is here $200 \mathrm{~N}$. The work time of the power cable tension is determined by calculating two options. In this paper we propose two options:

The strength of the tension arises immediately after the shot penetrator and is valid for the time of the landing process.
The strength of the cable tension arises under the condition of increasing the length of the rope, the rope starts to pull the unit at a height of one meter before the end of the time of landing.

In such cases, the operating mode of the clamping engines NV $=3$ is used. Now consider the case of landing in the presence of the cable tension force for all the time of landing. The results are shown in the following figures:
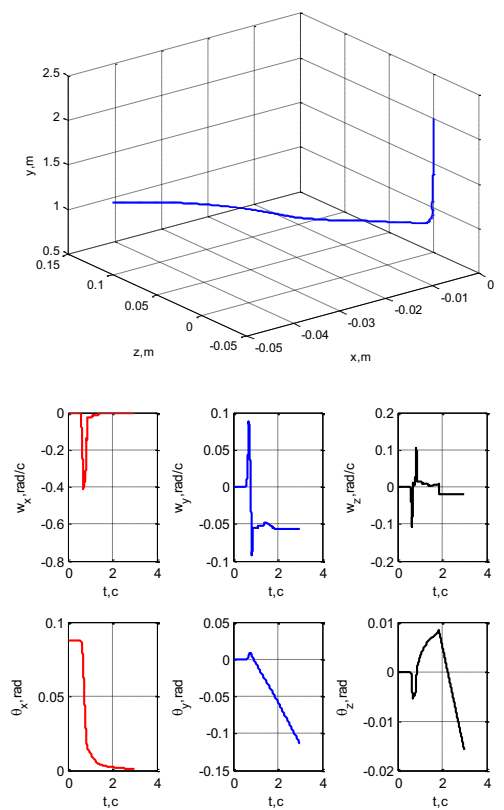

Figure 9. The cable operates at a height of one meter, $T=200 \mathrm{~N}$, $\mathrm{P}=200 \mathrm{~N}$

Figure 8 and Figure 9 showed that when the cable is valid for all the time of landing, it is possible to provide a more stable landing.

Now we will analyze the impact of the value of a cable tension on landing. Let the constant force of tension is $\mathrm{T}=300 \mathrm{~N}$ and $\mathrm{T}=400 \mathrm{~N}$. In both cases the cable is valid for all the time of landing. The results are shown in the following figures:
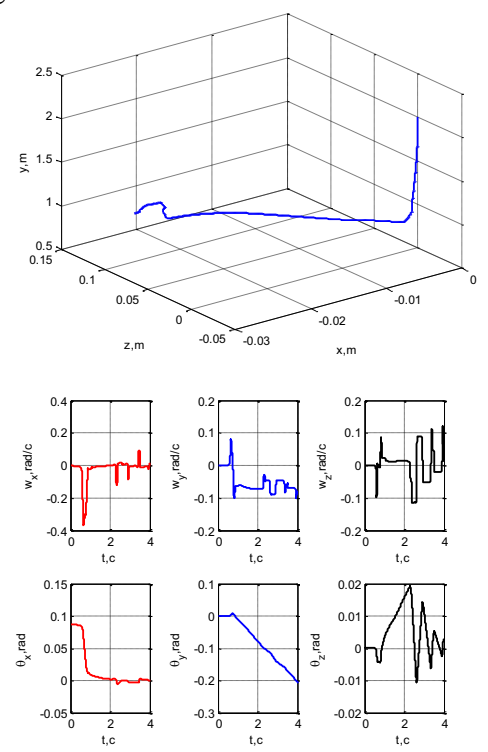

Figure 10. The cable works during all landing time, $\mathrm{T}=300 \mathrm{~N}$, $\mathrm{P}=200 \mathrm{~N}$ 

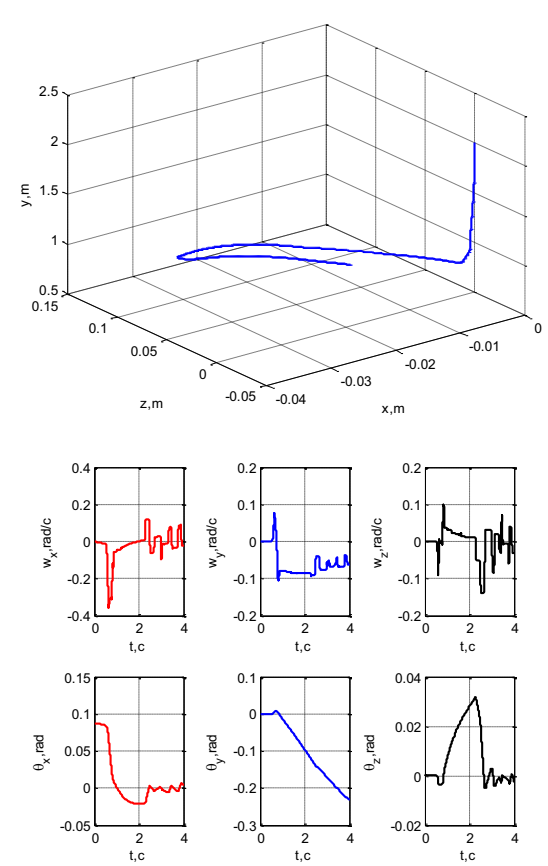

Figure 11. The cable works during all landing time, $T=400 \mathrm{~N}$, $\mathrm{P}=200 \mathrm{~N}$

From above presented calculation results it can be concluded that the amount of force the cable tension affects the landing. If the tension relatively small, the cable will not be able to pull the space lander to the surface of the celestial body, in the presence of enough tension force could ensure a successful landing.

\section{Conclusion}

The Analysis of results indicates that the complexation system (clamping engines and cable system) securing the space lander provides a successful landing on the surface of a small celestial body.

Thus, the most recommended conditions for landing on the surface of a small celestial body are such initial parameters specified as the action of a cable system for all landing time with a tension force $\mathrm{T}=400 \mathrm{~N}$, getting started clamping engine when the speed of the $\mathrm{Y}$-axis is positive. This work is important to find the optimal conditions of landing the space lander implementation and its future challenges. The successful landing of the space lander allows further, not only to study the dynamics of flight spacecraft, but also to develop the space program on the whole.

\section{References}

1. "Designing descent unmanned spacecraft" edited by Corresponding member of the Academy of Sciences of the USSR Kovtunenko V.M. M.: "Engineering" (1985)

2. V.P. Kazakoztsev, Chen Danhe, V.V. Koryanov, Method for calculation spacecraft longitudinal motion parameters during landing on the surface of a small celestial body. Herald of the Bauman Moscow State Technical University, series: Mechanical Engineering. 1 (2014) (94). p. 26-35

3. V.P. Kazakovtsev, V.V. Koryanov, Chen Danhe. Dynamics of landing a spacecraft on a celestial body with small field of gravity // 2015 5th international workshop on computer science and engineering: information processing and control engineering, WCSE 2015-IPCE (Information Processing and Control Engineering, Programme (2015) p. 191-195

4. V.P. Kazakovtsev, V.V. Koryanov, Chen Danhe. Investigation of the dynamics of the angular motion of the spacecraft when landing on a celestial body with a small field of gravitation. // Flight.10 (2014) p. 47-53

5. M. Hilchenbach, O. Kuechemann, H. Rosenbauer, Impact on a comet: Rosetta Lander simulations. Planetary and Space Science 48 (2000) p.361 \pm 369

6. D Steltzner Adam, K Nasif Annette. Anchoring Technology for In Situ Exploration of Small Bodies[C]//IEEE Aerospace Conference. Big Sky. Montana, USA, (2000) p.507-518

7. Zhijun Zhao, Research on the asteroid landing mechanism and its penetrating and anchoring. Dissertation for the Doctoral Degree in Engineering. Harbin Institute of Technology. (2014) p.2-25

8. E. Robert Lavender. Touchdown Dynamics Analysis of Spacecraft for Soft Lunar Landing. NASA Technical Note D-2001 (1964)

9. V.V. Koryanov, Method of calculating the descent of the spacecraft in the atmosphere using technology adaptation landing in different environmental conditions // Proceedings of the International Astronautical Congress, IAC 8 (2014) p.5628-5632

10. V.V. Koryanov, V.P. Kazakovtsev, Method for calculating the motion parameters of spacecraft. Natural and Technical Sciences. 9-10 (77) (2014) p. 179-184 